\title{
Determination of Sulfur Abundance in the Solar Wind
}

\author{
C. Giammanco $\cdot$ P. Bochsler · R. Karrer · F.M. Ipavich • \\ J.A. Paquette $\cdot$ P. Wurz
}

Received: 16 January 2007 / Accepted: 10 May 2007 / Published online: 5 July 2007

(C) Springer Science+Business Media B.V. 2007

\begin{abstract}
Solar chemical abundances are determined by comparing solar photospheric spectra with synthetic ones obtained for different sets of abundances and physical conditions. Although such inferred results are reliable, they are model dependent. Therefore, one compares them with the values for the local interstellar medium (LISM). The argument is that they must be similar, but even for LISM abundance determinations models play a fundamental role (i.e., temperature fluctuations, clumpiness, photon leaks). There are still two possible comparisons - one with the meteoritic values and the second with solar wind abundances. In this work we derive a first estimation of the solar wind element ratios of sulfur relative to calcium and magnesium, two neighboring low-FIP elements, using 10 years of CELIAS/MTOF data. We compare the sulfur abundance with the abundance determined from spectroscopic observations and from solar energetic particles. Sulfur is a moderately volatile element, hence, meteoritic sulfur may be depleted relative to non-volatile elements, if compared to its original solar system value.
\end{abstract}

Keywords Sun: abundances $\cdot$ Sun: solar wind

\section{Introduction}

The solar wind elemental composition is usually related to the solar atmosphere and to meteoritic abundances. Solar wind and meteoritic matter originate from the same nebula; however, fractionating processes could change the relative abundances. In particular, it has been observed that the low-FIP elements of the solar wind are enriched with respect to the photospheric abundances. Theories locate the fractionation process in the chromosphere, as a result of the interaction between neutrals, ionized atoms, and EUV radiation from

C. Giammanco $(\bowtie) \cdot$ P. Bochsler $\cdot$ R. Karrer $\cdot$ P. Wurz

Physikalisches Institut der Universität Bern, Sidlerstrasse 5, 3012 Bern, Switzerland

e-mail: giammanco@space.unibe.ch

F.M. Ipavich · J.A. Paquette

University of Maryland, College Park, MD 20742, USA 
the solar corona (e.g. Marsch et al. 1995). The low- and high-FIP elements are distinguished for FIP $<10 \mathrm{eV}$ and FIP $>10 \mathrm{eV}$, respectively. Sulfur is located in the transition $(\mathrm{FIP}=10.4 \mathrm{eV})$, therefore its reliable determination provides a good test for existing theories. We have ten years of data from the Mass Time Of Flight (MTOF) sensor of the CELIAS instrument (Hovestadt et al. 1995) on SOHO. In this paper we present a detailed analysis. The MTOF sensor response depends strongly on the wind speed, and for this analysis we select only time periods where the wind speed was in three velocity ranges, $380 \pm 20,390 \pm 20$, and $400 \pm 20 \mathrm{~km} / \mathrm{s}$. We accumulated the largest dataset in these velocity ranges. The velocity of the solar wind is provided from the Proton Monitor sensor and is given for protons (Ipavich et al. 1998).

MTOF can be conceptually divided into two parts: (1) an entrance system that makes a preselection in energy per charge and incident angle for the solar wind ions, which is governed by the $V_{\text {wave }}$ potential and (2) after a post-acceleration by a potential $V_{\mathrm{F}}$, the solar wind ions enter into a time-of-flight mass spectrometer, that measures their mass.

\section{The Spectra}

The MTOF sensor provides time-of-flight spectra (Fig. 1). The solar wind ions are usually multiply charged. However, after energy-per-charge selection, the ions are recharged to neutral, singly, or doubly ionized in the carbon foil of the MTOF sensor. The time-of-flight data are converted into mass-per-charge $(M / Q)$ spectra using a quadratic relation, since for a particle of mass $\mathrm{M}$ and charge $Q$ we have the relation: $M / Q=C\left(t-t_{\mathrm{o}}\right)^{2}$, where $t$ is the time of flight and $Q$ is the charge after charge exchange.

Each bin of the spectrum represents the number of particles at a given $M / Q$ and velocity range counted during the time of integration. To extract useful information we must take

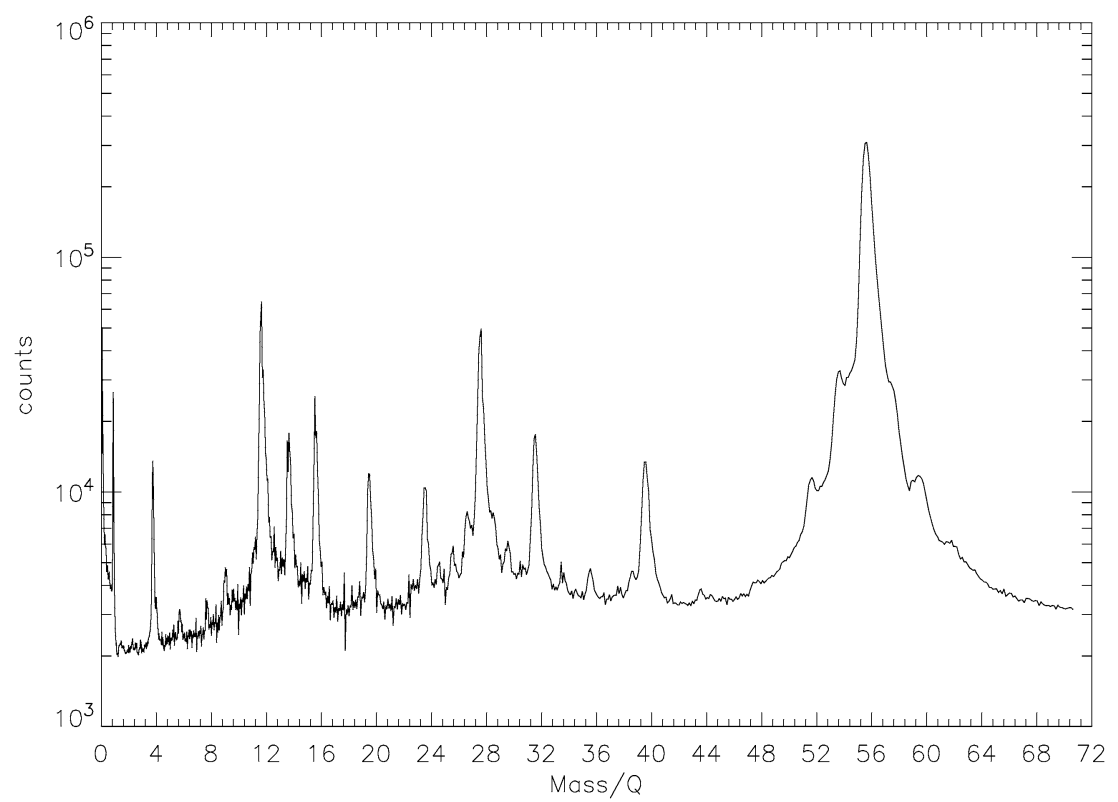

Fig. 1 Typical MTOF spectrum obtained for one of the three wind-speed interval integrated from year 1996 to 2006. The figure shows the peaks of $\mathrm{Fe} \mathrm{56,} \mathrm{S} \mathrm{32,} \mathrm{Ca} \mathrm{40,} \mathrm{Ni} \mathrm{58,} \mathrm{Ni} \mathrm{60,} \mathrm{and} \mathrm{Mg} 24$, among others 
into account the instrument response variation for the different mass-charge ratios and, if present, the contamination generated by doubly ionized elements.

\section{Response of the Entrance System}

The MTOF entrance system is a Wide Angle Variable Energy/Charge electrostatic analyzer (WAVE). Its function is to accept solar wind ions over a large energy-per-charge and incident-angle range. Its response is set through a $V_{\text {wave }}$ potential; however, it is different for each element and for each ionization state (Wurz 2001; Wurz et al. 1999). It also depends on the solar wind speed, the solar wind thermal speed, and the incident angle. In this work we suppose that the wind comes in the ecliptic plane from the Sun direction. The thermal speed is as a first approximation assumed to be equal to the value of the long time average of the Proton Monitor thermal speed measurement, i.e., 10 times less than the wind velocity. Figure 2 shows the effective area of the entrance system as function of the $V_{\text {wave }}$ voltage for different elements. For a more refined analysis in the future, we will use the actually measured thermal spreads provided by the proton monitor of CELIAS. To choose the best potential to determine a particular abundance ratio, it is convenient to analyze the ratio of the entrance system response for the two elements (Fig. 3).

Fig. 2 Response of the entrance system. The effective area is calculated taking into account a solar wind velocity of $380 \mathrm{~km} / \mathrm{s}$. For each element we interpolate a freezing temperature for this solar wind speed. Then we use the compilation of Mazzotta et al. (1998) to derive the respective charge ion distributions

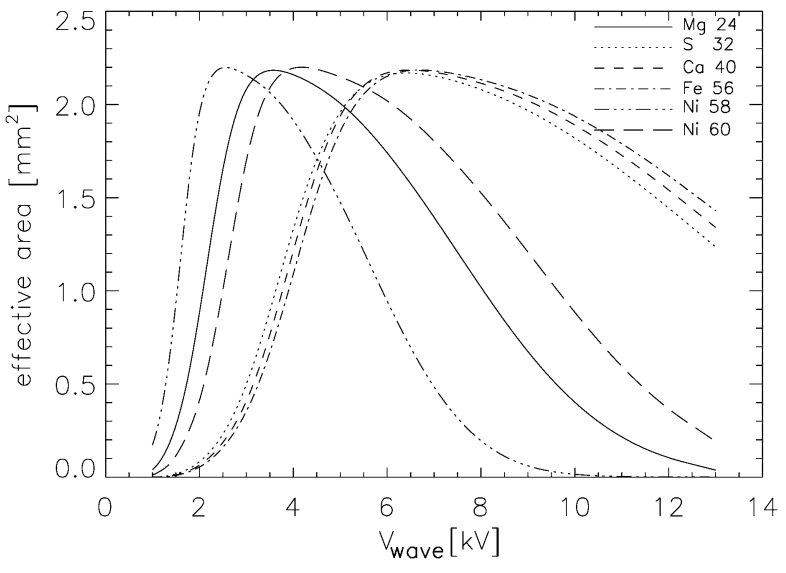

Fig. 3 Ratio of WAVE effective area for sulfur over magnesium, sulfur over calcium, and nickel over iron. The flat parts of the curves indicate the intervals of voltages that are best suited for the measurement of relative abundances

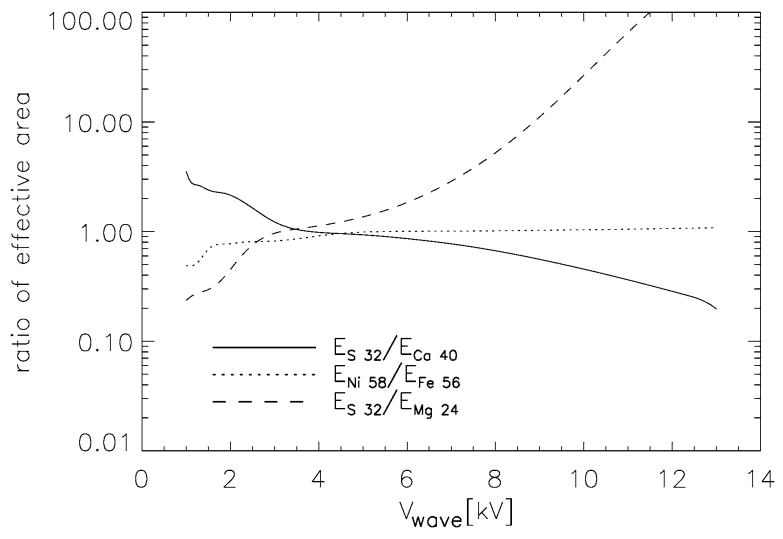


Fig. 4 Response of the mass spectrometer for different solar wind speeds in function of the post acceleration potential, $V_{\mathrm{F}}$. In the case of sulfur, the range of velocities that we have selected will be the principal source of the final uncertainty

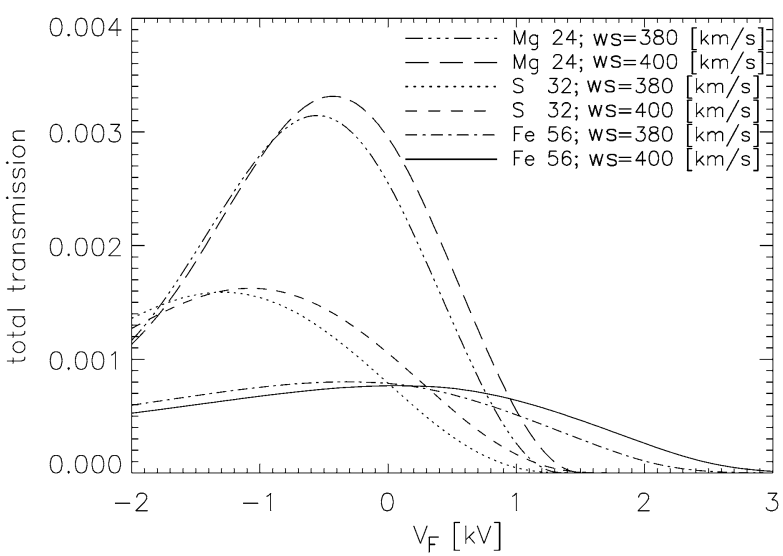

\section{Response of the Mass Spectrometer}

Once we have chosen the preferred voltage for the entrance system we must do the same for the spectrometer. The isochronous mass spectrometer measures the time of flight of incident particles. Its response depends on the element, the ionization state, and the incident angle (Fig. 4).

\section{The Measured Abundances}

To determine the ratio $[\mathrm{S}] /[\mathrm{Mg}]$ we have seven different combinations of voltage sets. One of them gives a $V_{\text {wave }}$ voltage far from the flat interval between 2 and $8 \mathrm{kV}$ (Fig. 3); thus, we exclude it from the analysis. For each accepted voltage set we calculate an abundance ratio assuming a Dirac distribution for the wind speed during the integration time, centered at 380,390 , and $400 \mathrm{~km} / \mathrm{s}$. Finally, we take the mean value of the calculated ratios as the final result. We do the same for the $[\mathrm{S}] /[\mathrm{Ca}]$ ratio. The results are shown in Table 1 .

Table 1 The reported ratios from other authors are derived by the respective abundances relative to hydrogen. The Ulysses/SWICS ratios are derived by the abundances relative to oxygen given in von Steiger et al. (2000) for solar maximum and minimum regimes of slow solar wind

$[\mathrm{S}] /[\mathrm{Mg}]$

$[\mathrm{S}] /[\mathrm{Ca}]$

Solar wind this work

Solar wind Ulysses/SWICS, $\max$

Solar wind Ulysses/SWICS, min

Photosphere Asplund et al. (2005)

Photosphere Grevesse and Sauval (1998)

Meteorites Anders and Grevesse (1989)

Meteorites Palme and Beer (1994)

SEP-derived corona Reames (1998)

SEP-derived corona Breneman and Stone (1985)

$\begin{array}{ll}0.36 \pm 0.16 & 4.6 \pm 1.0 \\ 0.33 \pm 0.10 & - \\ 0.36 \pm 0.11 & - \\ 0.41 \pm 0.06 & 6.8 \pm 0.6 \\ 0.56 \pm 0.09 & 9.3 \pm 1.2 \\ 0.49 \pm 0.03 & 8.5 \pm 0.7 \\ 0.42 \pm 0.03 & 7.1 \pm 0.5 \\ 0.162 \pm 0.005 & 3.00 \pm 0.13 \\ 0.142 \pm 0.099 & 1.44 \pm 0.03\end{array}$




\section{Conclusions}

In conclusion according to this first analysis, we infer that sulfur shows a small depletion in the solar wind relative to low-FIP elements, and that it behaves more like a low-FIP element despite a rather long ionization time in the chromosphere of $\sim 12$ seconds (Marsch et al. 1995). Our measurements are consistent with those of von Steiger et al. (2000).

Acknowledgements We acknowledge valuable suggestions by L.M. Blush and M. Iakovleva. This work was supported by the Swiss National Science Foundation

\section{References}

Anders, Grevesse, GCA 53, 197-214 (1989)

M. Asplund, N. Grevesse, A.J. Sauval, ASPC 336, 25 (2005)

H.H. Breneman, E.C. Stone, Astrophys. J. 299, L57 (1985)

N. Grevesse, A.J. Sauval, Space Sci. Rev. 5, 161 (1998)

D. Hovestadt et al., Sol. Phys. 162, 441 (1995)

F.M. Ipavich et al., J. Geophys. Res. 103, 17205 (1998)

F.M. Ipavich, J.A. Paquette, P. Bochsler, S.E. Lasley, P. Wurz, AIP Conf. 598, 101 (2001)

E. Marsch, R. von Steiger, P. Bochsler, Astron. Astrophys. 301, 261 (1995)

P. Mazzotta, G. Mazzitelli, S. Colafrancesco, N. Vittorio, Astron. Astrophys. Suppl. Ser. 133, 403 (1998)

H. Palme, H. Beer, in Astronomy and Astrophysics, vol. 3, ed. by Voigt (Springer, 1994), pp. 196-221

D.V. Reames, Space Sci. Rev. 5, 327 (1998)

R. von Steiger et al., J. Geophys. Res. 105, 27 (2000)

P. Wurz, Heavy ions in the solar wind: Results from SOHO/CELIAS/MTOF, University Bern, 2001

P. Wurz, M.R. Aellig, P. Bochsler, S. Hefti, F.M. Ipavich, A.B. Galvin, H. Grünwaldt, M. Hilchenbach, F. Gliem, D. Hovestadt, Phys. Chem. Earth 24, 421 (1999) 